\title{
INTERDISCIPLINARY APPROACH IN BONE METASTASES OF OVARIAN CANCER
}

doi: 10.2478/rojost-2018-0037

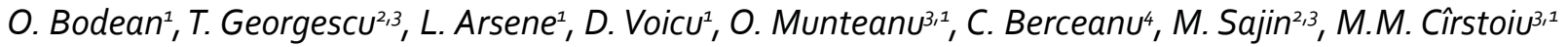
${ }^{1}$ Obstetrics and Gynecology Department, University Emergency Hospital, Bucharest, Romania ${ }^{2}$ Department of Morphopathology, University Emergency Hospital, Bucharest, Romania 3"Carol Davila" University of Medicine and Pharmacy, Bucharest, Romania ${ }^{4}$ Obstetrics and Gynecology Department, Craiova University of Medicine and Pharmacy, Craiova, Romania

Ovarian carcinoma is a deadly disease, with one of the highest case-to-fatality ratio amongst all gynecological malignancies. The high mortality of these tumors can be explained by the fact that most patients present at an advanced stage, with widely spread metastatic disease, especially within the peritoneal cavity. Extraperitoneal, occult metastases are usually rare in cancer surviving patients. Bone metastases are not a common finding, but their incidence seems to be higher than expected, as proven by autopsy studies. Because most clinicians are not very familiar with bone metastases of ovarian carcinoma, in this article we intended to discuss the most controversial aspects concerning the diagnosis of this type of disease.

Keywords: bone metastases, ovarian cancer, pathology 